\title{
Self-adjusting files in retreatment: a high-resolution micro-computed tomography study
}

Solomonov, Michael ; Paqué, Frank ; Kaya, Sadullah ; Adigüzel, Ozkan ; Kfir, Anda ; Yiğit-Özer, Senem

\begin{abstract}
INTRODUCTION: Rotary instruments that are used for retreatment are very effective, but most of them leave root filling residue in the canal. The aim of this study was to evaluate the efficacy of removing gutta-percha-based root fillings with ProTaper retreatment files (Dentsply Maillefer, Ballaigues, Switzerland) followed by F1 and F2 ProTaper instruments and to compare these results with those obtained with a 25 .06 ProFile instrument (Dentsply Maillefer) followed by the Self- Adjusting File (SAF; ReDent, Ra'anana, Israel) using high resolution micro-computed tomography (CT) scanning. METHODS: Twenty-eight mandibular molar teeth with oval distal root canals were divided into 2 equal groups of 14 teeth each. The distal root canals were instrumented with ProTaper files up to an F2 instrument, the roots were subsequently filled, and the root filling was allowed to set fully. Removal of the root canal filling was performed with D1-D3 ProTaper retreatment files followed by F1 and F2 ProTaper instruments or with a 25 .06 ProFile followed by SAFs. Chloroform was used in both groups to assist in the removal of the root filling material. High-resolution micro-CT scans were used to measure the residual quantities of the root filling material after completion of the procedures. Statistical analysis was performed using the Wilcoxon test and the Student t test. RESULTS: The median root filling residue in the ProTaper group was $5.39 \%$ (interquartile range $[\mathrm{IQR}]=4.71$ ) of the original volume of the root canal filling. In the ProFile and SAF group, the median residue was $0.41 \%$ (IQR $=1.64, \mathrm{P}<.001)$. An arbitrarily selected threshold of less than $0.5 \%$ residue was defined as "effectively cleaned," and $57 \%$ of the teeth treated with the ProFile and the SAF met this threshold, whereas none of the cases in the ProTaper group did. The ProFile and SAF procedure required less time than the ProTaper protocol. CONCLUSIONS: None of the retreatment methods rendered all of the canals completely free of all root filling residue. Under the conditions of this study, the ProFile and SAF procedure was more effective than the ProTaper procedure and left significantly less root filling residue in the root canal.
\end{abstract}

DOI: https://doi.org/10.1016/j.joen.2012.06.019

Posted at the Zurich Open Repository and Archive, University of Zurich

ZORA URL: https://doi.org/10.5167/uzh-71593

Journal Article

Accepted Version

Originally published at:

Solomonov, Michael; Paqué, Frank; Kaya, Sadullah; Adigüzel, Ozkan; Kfir, Anda; Yiğit-Özer, Senem (2012). Self-adjusting files in retreatment: a high-resolution micro-computed tomography study. Journal of Endodontics, 38(9):1283-1287.

DOI: https://doi.org/10.1016/j.joen.2012.06.019 


\section{Self-Adjusting Files in Retreatment: A High-Resolution Micro- Computed Tomography Study}

Michael Solomonov, DMD ${ }^{1}$, Frank Paqué, DMD², Sadullah Kaya, DMD ${ }^{3}$, Özkan Adıgüzel, DMD³ , Anda Kfir, DMD ${ }^{5}$, Senem Yiğit-Özer, DMD ${ }^{3}$

${ }^{1}$ Department of Endodontics, Sheba Hospital, Tel Hashomer, Israel

${ }^{2}$ Department of Preventive Dentistry, Peridontology and Cariology, University of Zürich, Zürich, Switzerland.

${ }^{3}$ Department of Endodontics, Faculty of Dentistry, University of Dicle, Diyarbakir, Turkey.

${ }^{4}$ Department of Endodontology, The Goldschleger School of Dental Medicine, Tel Aviv University, Tel Aviv, Israel.

Running Title: The use of SAF in retreatment

Keywords: micro-CT, ProTaper, Retreatment, SAF

\section{Corresponding Author:}

Michael Solomonov, DMD

13/54 Ha-Kfar St,

Kiryat Ono, 55525

Israel

mishasol@gmail.com 


\section{Abstract}

Introduction: The aim of this study was to evaluate the efficacy of removing root fillings with ProTaper retreatment files followed by F1 and F2 ProTaper instruments and to compare these results with those obtained with a \#25.06 ProFile instrument followed by the Self- Adjusting File (SAF), using high resolution micro-CT.

Methods: Twenty-eight mandibular molar teeth with oval distal root canals were divided into two equal groups of 14 teeth each. The distal root canals were instrumented with ProTaper files up to an F2 instrument, the roots were subsequently filled, and the root filling was allowed to set fully. Removal of the root canal filling was performed with D1-D3 ProTaper retreatment files followed by F1 and F2 ProTaper instruments or with a \# 25 .06 ProFile followed by SAFs. Chloroform was used in both groups to assist in removal of root filling material. High-resolution micro-CT was used to measure the residual quantities of root filling material after completion of the procedures. Statistical analysis was performed using the Wilcoxon test and Student's ttest. Results: The median root filling residue in the ProTaper group was $5.39 \%$ (IQR 4.71) of the original volume of the root canal filling. In the ProFile and SAF group, the median residue was $0.41 \%($ IQR 1.64) $(p<0.001)$. An arbitrarily selected threshold of less than $0.5 \%$ residue was defined as "clean", and $57 \%$ of teeth treated with the ProFile and SAF met this threshold, while none of the cases in the ProTaper group did. The ProFile and SAF procedure required less time than did the ProTaper protocol. Conclusions: None of the retreatment methods rendered all of the canals completely free of 
all root filling residue. The ProFile and SAF procedure was more effective than the ProTaper procedure and left significantly less root filling residue in the root canal. 


\section{Introduction}

The initial stage of any retreatment procedure is removal of previous root filling material to allow adequate cleaning, disinfection and obturation of the root canal space $(1,2)$. This task can easily be accomplished with rotary files, which allow removal of the bulk of root filling material within a few minutes (39). However, recent studies have indicated that such a procedure performed on its own leaves a significant amount of root filling residue along the canal wall $(4,10-12)$. This finding may partly explain the low reported success rate of retreatment in cases with post-treatment disease (13).

In straight canals, an operating microscope can be used to evaluate and facilitate removal of root filling residues. In contrast, curved canals limit the view beyond the curvature, and the operator has to rely on radiographs(12), which have recently been shown to be unreliable and misleading for such evaluations $(7,11)$.

Recently, Abramovitz et al. have tested the efficacy of the Self-Adjusting File system in removing root filling residue that had been left in curved canals after using ProTaper retreatment files (12). After removal of the bulk of filling material, a drop of chloroform was placed in the canal, and the SAF was used for 1 min without irrigation followed by 3 min with sodium hypochlorite irrigation, resulting in much cleaner canals than when only rotary files were used. 
The present study extended these observations to include oval canals in the distal roots of mandibular molars. Rotary instruments may effectively remove root fillings from straight, narrow canals with round cross-sections. However, for oval canals, rotary instruments are likely to be less effective, as indicated by their inability to shape oval canals (14). We assumed that because the SAF is supposed to adapt to the cross-section of oval canals and exert a scrubbing effect on the canal walls $(15,16)$, the SAF may also perform well during retreatment under these challenging conditions.

In the aforementioned study (12), planar 2D radiographs were used to evaluate the results. Recent studies have demonstrated the limitations of 2D radiographs for these types of evaluations $(7,11)$; therefore, we used a highresolution micro-CT instead. It has recently been shown that this method can easily be adapted to differentiate between canal walls and root filling material (17). Additionally, this method enables three-dimensional evaluation of the entire canal and avoids any potential operator bias during interpretation of the results.

The present study was designed to evaluate the efficacy of removing root fillings with ProTaper retreatment files followed by F1 and F2 ProTaper instruments and to compare these results with those obtained with a \#25.06 ProFile instrument followed by the Self-Adjusting File (SAF), using high resolution micro-CT.

\section{Materials and Methods}

\section{Selection of teeth}


Forty mandibular molars were selected from a random collection of teeth that had been extracted for periodontal reasons. Initial inclusion criteria were single distal root canal, no previous root canal treatment, straight roots of similar length and completely developed apices.

Specimens were subsequently mounted on SEM carriers (014001-T, Bal-Tec AG, Balzers, Liechtenstein) and pre-scanned using a high-resolution microcomputed tomography system ( $\mu \mathrm{CT} 40$, Scanco Medical, Brüttisellen, Switzerland) with an isotropic resolution of $20 \mu \mathrm{m}$ at settings of $70 \mathrm{kV}$ and 114 $\mu \mathrm{A}$. Based on the root canal cross-sections of the initial scans, the teeth were divided into two groups using the flatness of the canal as the key parameter. The ratio of the bucco-lingual to the mesio-distal diameter of the canal was calculated $6 \mathrm{~mm}$ from the root tip. Teeth with a ratio of between 1.4 and 2.8 were included. Teeth were pair-matched based on this parameter and subsequently one tooth of each pair was randomly assigned to one of the two projected experimental groups while the other was assigned to the other group. The two resulting groups consisted of 14 teeth each that fitted all of the above-mentioned inclusion criteria. Assignment of the procedure to the two groups was made by the flip of a coin, after the groups had been formed, and after an experienced operator had performed root fillings on all teeth (see below). The operator was blinded to the group assignments.

\section{Root canal preparation and filling}

All teeth were subjected to chemo-mechanical preparation of the distal root. An access cavity was prepared in each tooth and C+ files (DentsplyMaillefer, Ballaigues, Switzerland) were used to negotiate the canal, and 
working length was established $1 \mathrm{~mm}$ short of the apical foramen. ProTaper files (Dentsply-Maillefer) were used to prepare the canals and were operated with VDW Gold motor (VDW GmbH, Munich, Germany) at a speed of $150 \mathrm{rpm}$ and a torque of $300 \mathrm{Ncm}$. The coronal orifices of the distal canals were enlarged using ProTaper SX files (Dentsply-Maillefer, Ballaigues, Switzerland), which were inserted to a depth of $5 \mathrm{~mm}$. Subsequently, S1 and S2 files were used to working length and followed by F1 and F2, which were used to working length, as well. The Sx, S1 and S2 were operated with a brushing motion according to the shape of the canals. A syringe with a $27 \mathrm{G}$ needle was inserted to $2 \mathrm{~mm}$ short of the entire working length, and the canal was irrigated with $1 \mathrm{~mL}$ of sodium hypochlorite after each instrumentation. The canals were subsequently irrigated with $2 \mathrm{~mL}$ of $17 \%$ EDTA and were dried using paper points.

Root filling was performed with tapered gutta-percha master cones (ProTaper, Dentsply-Maillefer) and AH-Plus sealer (Dentsply-DeTrey, Konstanz, Germany) following the lateral compaction method. A lentulo spiral was used to fill the canal with sealer. After insertion of the master cone, accessory gutta-percha cones were added with a \#25 finger spreader (DentsplyMaillefer). Any excess of gutta-percha was removed with a hot excavator at the level of the canal orifice. The sealer was allowed to set for 30 days at $37^{\circ}$ C and $100 \%$ humidity.

All teeth were subsequently scanned again, and the volume of root filling was determined (see below).

\section{Retreatment: ProTaper}


The retreatment procedure consisted of two stages. First, the bulk of the root filling material was removed using ProTaper retreatment instruments, which was followed by removal of the remaining material using the F1 and F2 ProTaper files.

Stage 1: ProTaper Universal retreatment files D1-D3 (Dentsply-Maillefer) were used to remove the root filling material. The files were operated with a VDW Gold motor with a torque of $300 \mathrm{Ncm}$ and a rotation speed of $180 \mathrm{rpm}$ for D1 and $150 \mathrm{rpm}$ for D2 and D3. D1 was used from coronal to the middle thirds until no debris was visible on the file surface when it was removed from the canal (18). A drop of chloroform (10 $\mu \mathrm{l})$ was placed in the canal, and the D2 instrument was used to $2 \mathrm{~mm}$ short of working length. Next, the canal was irrigated with $2 \mathrm{~mL}$ of $5.25 \% \mathrm{NaOCl}$ followed by $2 \mathrm{~mL}$ of $17 \%$ EDTA, which were delivered with a syringe and a $27 \mathrm{G}$ needle. The canal was subsequently dried with paper points, and another drop of $10 \mu \mathrm{L}$ of chloroform was placed in the canal. Next, the D3 instrument was inserted to working length using the above mentioned endpoint criterion. Subsequently, the canal was irrigated with $2 \mathrm{~mL}$ of $5.25 \% \mathrm{NaOCl}$ followed by $2 \mathrm{~mL}$ of $17 \%$ EDTA.

Stage 2: F1 and F2 ProTaper files were used with an added brushing motion because of the shape of the canal. Irrigation with $1 \mathrm{~mL}$ of $5.25 \% \mathrm{NaOCl}$ and 1 $\mathrm{mL}$ of $17 \%$ EDTA was performed after each instrumentation. We used $20 \mu \mathrm{l}$ of chloroform, $8 \mathrm{~mL}$ of $5.25 \% \mathrm{NaOCl}$ and $8 \mathrm{~mL}$ of $17 \%$ EDTA for the entire procedure. The time required to accomplish this procedure was recorded, using a stopwatch. 
The canals were subsequently washed with $2 \mathrm{~mL}$ of distilled water, dried with paper points and stored at $100 \%$ humidity at room temperature. The retreatment procedure was performed by an operator who had extensive clinical experience with this type of procedure (SYO). The retreatment files were replaced after every two retreatment procedures. Next, teeth were scanned again, and the volume of the remaining radiopaque residue was determined (see below).

\section{Retreatment: ProFile and SAF}

The retreatment procedure consisted of two stages. First, the bulk of root filling material was removed with the ProFile instrument, which was followed by removal of any remaining material using the SAF with sodium hypochlorite and chloroform.

Stage 1: A ProFile rotary file (\#25 with a .06 taper, $25 \mathrm{~mm}$, Dentsply-Maillefer) was used to remove the bulk of the root filling material $(3,5)$. It was operated with an X-Smart motor at a torque of $2.4 \mathrm{Ncm}$ and a speed of $600 \mathrm{rpm}$. Pecking and brushing motions were applied to remove the gutta-percha down to working length.

Stage 2: After removal of the bulk of the root filling material, the Self-Adjusting File (SAF, 2.0 mm diameter, ReDent, Ra'anana, Israel) was used. The SAF was operated with an X-Smart motor (Densply-Maillefer) adapted with a 1:1 gear (NSK, Tochigi, Japan) and a vibrating RDT3-NX hand-piece head (ReDent). The rotation speed was set to $5000 \mathrm{rpm}$, which resulted in 5000 inand-out vibrations per min with an amplitude of $0.4 \mathrm{~mm}$. Using a VATEA peristaltic pump (ReDent), which was connected to the hollow SAF via a 
silicone tube, continuous irrigation was applied when indicated. $\mathrm{A} 4 \% \mathrm{NaOCl}$ solution was used with a flow rate of $4 \mathrm{~mL} / \mathrm{min}$.

First, the SAF was operated in the canal for 1 min under sodium hypochlorite irrigation to remove coarse particulate material. Next, the canal was dried with paper points and filled with $10 \mu \mathrm{L}$ of chloroform before the SAF was operated in the canal for an additional minute without any irrigation (the VATEA pump turned off). Subsequently, the canal was refilled with $10 \mu \mathrm{L}$ of chloroform, and the SAF was operated in it again for $1 \mathrm{~min}$. Next, the pump was turned on, and the SAF operated with sodium hypochlorite irrigation for 30 seconds. The canal was subsequently flushed with $1 \mathrm{ml}$ of $17 \%$ EDTA, which was left in the canal while the SAF operated in it for another 30 seconds with the pump turned off. A total of $20 \mu \mathrm{l}$ of chloroform, $1 \mathrm{~mL}$ of $17 \%$ EDTA and $6 \mathrm{~mL}$ of $\mathrm{NaOCl} 4 \%$ was used for the entire procedure. The time required for this procedure was recorded using a stopwatch.

The canals were subsequently washed with $2 \mathrm{~mL}$ of distilled water, dried with paper points and stored at $100 \%$ humidity at room temperature. This twostage retreatment procedure was performed by an operator who had extensive clinical experience with this type of procedure (MS). The teeth were scanned again (see below), and the volume of the remaining radiopaque residue was determined.

\section{Micro-CT measurements and evaluations}

Using a commercially available micro-computed tomography system ( $\mu$ CT 40, Scanco Medical), three high-resolution scans were performed per tooth at the following time points: (a) after canal instrumentation, (b) after root filling and 
(c) after the retreatment procedure. Teeth were scanned at $70 \mathrm{kV}$ and $114 \mu \mathrm{A}$ with an isotropic resolution of $20 \mu \mathrm{m}$, resulting in 600 to 800 slices per root. High resolution scans after root canal filling and retreatment procedures were run with five-fold integration time to reduce the noise and the scattering effect provoked by radiopaque root filling materials. The volume of interest was selected extending from the distal root canal orifice to the apex of the root. After determining the region of interest all teeth were three-dimensionally (3D) reconstructed. Based on the different radiopacity of root dentin, gutta-percha and the AH Plus sealer used in the current study, it was possible to differentiate between these entities on $\mu C T$ scans (19). Grey scale ranges were determined for each material based on individual scans. To visualize the different materials, these were depicted in false colors in the 3D reconstructions of the $\mu$ CT scans using specific software (VGStudio Max 2.1, Volumegraphics, Heidelberg, Germany).

Volumes of root fillings before and after the retreatment procedures were calculated using specially developed software (IPL V5.06B, Scanco Medical).

\section{Data presentation and statistical analysis}

Data pertaining to canal shape after preparation were expressed as the ratio of the bucco-lingual and mesio-distal canal diameter at $6 \mathrm{~mm}$ coronal of the root tip. Filled root canal volume was expressed in $\mathrm{mm}^{3}$. These data were normally distributed (Shapiro-Wilk test), and are thus presented as means \pm standard deviations.

Remaining root filling volumes after the different retreatment procedures were expressed both in $\mathrm{mm}^{3}$ and as $\%$ of the total initial root filling volume. These 
data were skewed and therefore were compared using the Wilcoxson test and presented as median values and inter-quartile ranges (IQR). For all statistical analyses a commercially available computer program (JMP, SAS Institute Inc., Cary, NC, USA) was used with the alpha-type error set at 5\% $(P<0.05)$. In addition, another clinically oriented parameter was used and arbitrarily defined canals as "clean" when less than $0.5 \%$ of root filling residue was left in the canal. 


\section{Results}

The initial ratio of the bucco-lingual diameter to the mesio-distal diameter of the canal was $1.93 \pm 0.38$ in the ProTaper group and $2.03 \pm 0.49$ in the ProFile and SAF group. The difference was not statistically significant. The mean volumes of the root filling were $6.86 \pm 1.60 \mathrm{~mm}^{3}$ and $7.89 \pm 2.34 \mathrm{~mm}^{3}$ in the ProTaper group and the ProFile and SAF group, respectively. The groups did not differ from each other in either of these parameters (Student's t-test, $p>0.05)$. Therefore, a similar level of potential challenge was presented to each of the two retreatment protocols.

The mean time required to complete the ProTaper retreatment protocol was $10.1( \pm 0.3) \mathrm{min}$ with a range of 8.9 to $11.9 \mathrm{~min}$. The mean time required to complete the ProFile/SAF protocol was $4.8( \pm 0.1) \mathrm{min}$ with a range of 4.5 to $5.0 \mathrm{~min}(\mathrm{p}<001)$. Of the total time required for the ProTaper protocol, the instrumentation alone lasted $5.5( \pm 0.2) \mathrm{min}$, while the irrigation required 4.6 $( \pm 0.2) \mathrm{min}$. These two procedures could not be timed individually in the second group because instrumentation and irrigation were performed simultaneously.

None of the procedures removed all the remains of the root canal filling in all teeth. The volume of root filling residue in both groups did not have a normal distribution, and the data were skewed in both groups (Shapiro-Wilk test). The median volume of the root filling residue was $0.38 \mathrm{~mm}^{3}$ (IQR 0.43 ) and 0.03 
$\mathrm{mm}^{3}$ (IQR 0.14) in the ProTaper retreatment and ProFile and SAF group, respectively (Wilcoxon test, $p<0.001$ ).

These residue volumes represented 5.39\% (IQR 4.71) and 0.41\% (IQR 1.64) of the original root filling volume, respectively (Wilcoxon test $p<0.001$ ) (Figure 1). Representing reconstructed images of both groups are presented in Figure 2.

Among the roots in the ProFile and SAF group, 57\% presented with "clean" canals, i.e., the root filling residue was less than $0.5 \%$ of the original root filling volume. In contrast, none of the teeth in the ProTaper group fell into this category.

\section{Discussion}

None of the retreatment methods that were tested in the present study could render all canals completely free of root filling residues. However, the ProFile and SAF protocol left significantly less residue and required less time than did the ProTaper retreatment protocol (Figure 1,2).

The two protocols were compared based on a single parameter, which was their ability to remove the root filling without enlarging the root canal, particularly in the apical part. The canal was originally prepared to working length with an F2 ProTaper instrument. Therefore the D3 instrument, with its size 20 tip, could not be expected to completely remove the apical part of the root filling The manufacturer's instructions for the ProTaper retreatment files were followed with some modifications. F1 and F2 instruments were used to 
remove the remaining root filling material in the apical section after the D3 instrument had been used.

The ProFile and SAF protocol was a modification of a protocol recently suggested and studied by Abramovitz et al. (12). A size 25 ProFile instrument with a .06 taper was used, as its apical part is similar in size to that of the F2 ProTaper (size 25$)$ with a smaller taper (.06 compared to .08, respectively). Each of the protocols consisted of a two-stage procedure. In both procedures, the aim of the first stage was to remove the bulk of root filling material to working length. The aim of the second stage was to remove the root filling residue remaining in the canal.

Several studies have shown that retreatment with either ProFile or ProTaper retreatment files alone failed to render the canals free of residue $(4,5,10-12)$. When Abramovitz et al. used only ProTaper retreatment files in curved canals of mesial roots of mandibular molars, they found large amounts of residual root filling material in the apical third of the canals. This result should have been expected because these researchers initially prepared the apical size of the canal to a size of $30-45$, while the tip of the D3 retreatment file has a size of 20 with a .07 taper. Therefore, the manufacturer's instructions for the ProTaper indicate that whenever the apical diameter of the canal is larger than the tip of the D3 retreatment file, the root filling material that is left in the canal after its use should be removed with a different instrument. In the present study, this procedure was performed in the ProTaper group using F1 and F2 ProTaper files (apical size 20 with .07 taper and apical size 25 with .08 taper, respectively), which constituted the second stage of the ProTaper retreatment procedure. Larger ProTaper files, such as the F3 (apical size 30 
with a .09 taper), were avoided for the following reasons: (a) they may alter the shape of the canal, which was not the aim of this study, (b) when using such a stiff instrument, one may run the risk of canal transportation (20) and (c) it has recently been suggested that such procedures may increase the incidence of micro-cracks in the root dentin $(21,22)$.

The bulk of the root filling material was removed in the other group using ProFile instruments with an apical size 25 and a .06 taper. This approach was used to maintain the original dimensions of the canal, as explained above. The second stage in this group was performed with an SAF instrument. The SAF is supposed to adapt to the cross-section of the canal and to have a scrubbing effect on the canal walls $((15,16,23,24)$. However, it has been shown by Peters and Paqué (25) that in large canals, the 1.5-mm diameter SAF was less effective than the SAF with a 2.0-mm diameter. Therefore, in the present study, an SAF with a $2.0-\mathrm{mm}$ diameter was selected instead of one with a 1.5-mm diameter, which was used by Abramovitz et al. (12). The present results indicate that the retreatment protocol of ProFile size25 .06 followed by $2.0-\mathrm{mm}$ SAF was more effective than that of ProTaper retreatment files followed by ProTaper F1 and F2 instruments. This approach required less time to accomplish the procedure and resulted in cleaner canals, as defined by the amount of residual root filling material. Furthermore, based on our arbitrary definition, more cases in the ProFile and SAF group could be called "clean" (having less than $0.5 \%$ residue) than in the ProTaper group. .

The present study differs from that of Abramovitz et al.(12), because the latter used planar 2D radiographs to evaluate the results. Kfir et al. (11) recently 
reported that such radiographs fail to represent the real cleanliness of the canals. The gold standard for evaluation remains direct visualization by microscopy. Therefore, the current study utilized 3D high-resolution micro-CT as the evaluation tool. This tool has previously been used to evaluate the 3D quality of root canal fillings (17).

\section{Conclusions}

1. Neither retreatment protocol could render all canals completely free of root filling residues.

2. Retreatment with ProFile \#25 .06 followed by SAF resulted in less residual root filling material than did retreatment with ProTaper retreatment files followed by ProTaper F1 and F2 instruments.

3. The ProFile and SAF protocol required less time than did the ProTaper protocol.

4. A higher number of "clean" canals were found in the ProFile and SAF group than in the group treated with ProTaper retreatment files. None of the teeth in the ProTaper retreatment file group fell into this category.

Note: This manuscript was English edited by American Journal Experts 


\section{References}

1. Bergenholz G, Lekholm U, Milthorn R, Heden G, Odesjo B, Ergostrom B. Retreatment of endodontic fillings. Scand J Dent Res 1979;87:217-24.

2. Stabholz A, Friedman S. Endodontic retreatment-case selection and technique, Part 2: Treatment planning for retreatment. J Endod 1988;14: 60714.

3. Sae- Lim V, Rajamanickam I, Lim BK, Lee HL. Effectiveness of ProFile .04 taper rotary instruments in endodontic retreatment. J Endod 2000;26:100-4.

4. Imura N, Kato AS, Hata G-I, Uemura M, Toda T, Weine F. A comparison of the relative efficacies of four hand and rotary instrumentation techniques during endodontic retreatment. Int Endod J 2000;33:361-6.

5. Baratto Filho F, Ferreira EL, Fariniuk LF. Efficiency of the 0.04 taper ProFile during the re-treatment of gutta-percha-filled root canals. Int Endod J 2002;35:651-4.

6. Hülsmann M, Bluhm V. Efficacy, cleaning ability and safety of different rotary $\mathrm{NiTi}$ instruments in root canal retreatment. Int Endod J 2004;37:468-76. 
7. de Carvalho Maciel AC, Zaccaro Scelza MF. Efficacy of automated versus hand instrumentation during root canal retreatment: an ex vivo study. Int Endod J 2006; 39:779-84.

8. Gu L-S, Ling J-Q, Wei X, Huang X-Y. Efficacy of ProTaper Universal rotary retreatment system for gutta-percha removal from root canals.Int Endod $\mathrm{J}$ 2008;41:288-95.

9. Taşdemir T, Er K, Yildirim T, Çelik D. Efficacy of three rotary NiTi instruments in removing gutta-percha from root canals. Int Endod J 2008; 41: 191-6.

10. Saad AY, Al-Hadlaq SM, Al-Katheeri NH. Efficacy of two rotary NiTi instrunents in the removal of gutta-percha during root canal retreatment. $\mathrm{J}$ Endod 2007;3:38-41.

11. Kfir A, Tsesis I, Yakirevich E, Matalon S, Abramovitz I. The efficacy of five re-treatment techniques :microscopic vs. radiographic evaluation. Int Endod J 2012;45:35-41.

12. Abramovitz I, Relles-Bonar S, Baransi B, Kfir A. The effectiveness of a self-adjusting file to remove residual gutta-percha after retreatment with rotary files. Int Endod J 2012;(in press) 
13. de Chevigny C, Dao TT, Basrani BR, Marquis V, Farzaneh M, Abitbol S, Friedman S. Outcome in endodontics: The Toronto Study-Phases 3 and 4: orthograde retreatment. J Endod 2008;34:131-7.

14. De-Deus G, Souza EM, Barino B, Maia J, Zamolyi RQ, Reis C, Kfir a. The self-adjusting file optimizes debridment quality in oval-shaped root canals. J Endod 2011;37:701-5.

15. Metzger Z, Teperovich E, Zary R, Cohen R, Hof R. Respecting the root canal: a new concept of a self-adjusting file (SAF). J Endod 2010; 36: 679-90.

16. Hof R, Perevalov V, Eltanani M, Zary R, Metzger Z. The self-adjusting file (SAF). Part 2: Mechanical analysis. J Endod 2010; 36: 691-6.

17. Metzger Z, Zary R, Cohen R, Teperovich E, Paqué F. The quality of root canal preparation and root canal obturation in canals treated with rotary versus self-adjusting files: a three-dimensional micro-computed tomographic study. J Endod 2010; 36:1569-73.

18. Schirrmeister JF, Hermanns P, Meyer KM, Goetz F, Hellwig E. Detectability of residual Epiphany and gutta-percha after root canal retreatment using a dental operating microscope and radiographs: an ex vivo study. Int Endod J 2006;39:558-65 
19. Jung $M$, Lommel $D$, Klimek J. The imaging of root canal obturation using micro-CT. Int Endod J 2005;38:617-26.

20. Kunert GG, Camargo VR, Fontanella VRC, de Moura AAM, Barletta FB. Analysis of apical root transportation associated with ProTaper Universal F3 and F4 instruments by using digital subtraction radiography. J Endod 2010;36: 1052-5.

21. Shemesh H, Roeleveld AC, Wesselink PR, Wu MK. Damage to root dentin during retreatment procedures. J Endod. 2011;37:63-6.

22. Adorno CG, Yoshioka T, Suda H. Crack initiation on the apical root surface caused by three different Nickel-Titanium rotary files at different working lengths. J Endod 2011;37:522-5.

23. Peters OA, Paqué F. Root canal preparation of maxillary molars with the Self-adjusting File:A micro-computed tomography study. J Endod 2011;37: 53-7.

24. Versiani MA, Pécora JD, de Sousa-Neto MD.Flat-oval root canal preparation with Self-Adjusting File instrument: A micro-computed tomography study. J Endod 2011; 37:1002-7. 
25. Peters OA, Boessler C, Paqué F. Root Canal Preparation with a Novel Nickel-Titanium Instrument Evaluated with Micro-computed Tomography: Canal Surface Preparation over Time. J Endod 2010;36:1068-72. 


\section{Legends to Figures}

Figure 1. Percent of residual root filling material that was left in the canal after retreatment. Percent of residual root filling material that was left in the canal after removal with ProTaper retreatment files followed by F1 and F2 ProTaper files compared to removal with ProFile followed by SAF.

Figure 2. Representative cases of root filling removal.

In the three-dimensional reconstructions, gutta-percha is depicted in pink and the sealer in light-brown.

(A) Root filling residue that remained in the canal after retreatment with ProTaper. Left, the canal after preparation; center, the canal filling after obturation; right, the root filling residue left in the canal after retreatment with ProTaper.

(B) Root filling residue that was left in the canal after retreatment with Profile and SAF. Left, the canal after preparation; center, the canal filling after obturation; right, the root filling residue left in the canal after retreatment with ProFile and SAF. 


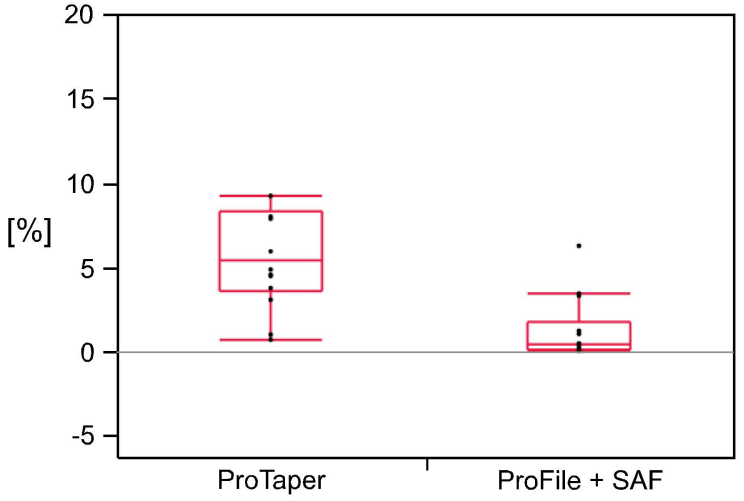


